\title{
Encephalopathy, CTCAE 5.0
}

National Cancer Institute

\section{Source}

National Cancer Institute. Encephalopathy, CT CAE 5.0. NCI Thesaurus. Code C146699.

A disorder characterized by a pathologic process involving the brain. 